\section{LAPAROSCOPY AND VIDEO ASSISTED THORACOSCOPY FOR INTERVAL DEBULKING SURGERY IN OVARIAN CANCER}

C Yelo Docio*, J De Santiago Garcia, M Fernandez Chereguini, S Alonso, A Conde Adan. MD Anderson Cancer Center Madrid, Madrid, Spain

\subsection{6/ijgc-2021-ESG0.517}

Introduction/Background* Minimal invasive surgery for interval debulking surgery for stage IV ovarian cancer

- 52 years old

- CT\&MRI - $11 \mathrm{~cm}$ adnexal mass and peritoneal

carcinomatosis

- PET scan:

- Left pleural effusion.

- Increased pleural FDG uptake

- Giant hypermetabolic adnexal mass

Methodology Video recorded surgery

Result(s)* Optimal cytorreduction after 3 cycles of chemotherapy.

Conclusion* Minimally invasive surgery may be considered for the management of patients with advanced ovarian cancer who have undergone neoadjuvant chemotherapy

\section{RELAXIN AS A POTENTIAL DIAGNOSTIC BIOMARKER FOR OVARIAN CANCER- A PROSPECTIVE STUDY}

${ }^{1} \mathrm{~F}$ Gkrozou, ${ }^{2} \mathrm{C}$ Pappa*${ }^{*}{ }^{3} \mathrm{O}$ Tsonis, ${ }^{4} \mathrm{E}$ Dimitriou, ${ }^{5} \mathrm{M}$ Paschopoulos. ${ }^{1}$ University Hospitals Birmingham NHS Foundation Trust, Obstetrics and Gynaecology, Birmingham, UK; ${ }^{2}$ Oxford University Hospitals, NHS Foundation Trust, Gynaecological Oncology , Oxford, UK; ${ }^{3} S t$ Bartholomew's Hospital, Barts Health NHS, Centre for Reproductive Medicine, London, UK; ${ }^{4}$ National and Kapodistrian University of Athens, Medical School and Department of Mathematics , Athens, Greece; ${ }^{5}$ University Hospital of loannina, Obstetrics and Gynaecology, loannina, Greece

\subsection{6/ijgc-2021-ESG0.518}

Introduction/Background* Ovarian cancer is a leading cause of female mortality worldwide. Although novel approaches on this disease have been developed, overall survival rates remain moderate due to the lack of scientific evidence promoting screening at early stages of the disease. A number of biomarkers have been suggested as predictive for this type of cancer.In this study we aimed to understand the role of relaxin in different types of ovarian cancer and to assess its diagnostic and prognostic significance.

Methodology A total of eighty one (81) patients with diagnosed ovarian cancer, one hundred and four (104) women, with indication of benign ovarian cyst after appropriate imaging investigation and fourty four (44) healthy women, used as control, have been recruited. Blood samples were collected prospectively, just before the operation. We detected the levels of relaxin, $\mathrm{Ca} 125$ and HE-4 and we calculated the Risk of Ovarian Malignancy Algorithm (ROMA). The outcomes of using relaxin, ca125, HE4 and ROMA values as biomarkers were compared in cases of ovarian cancer, healthy women and women with benign masses to identify the statistical importance of each marker.

Result(s)* In our study the levels of relaxin were significantly higher in cases of ovarian cancer, when they are compared with cases of benign ovarian cysts or in healthy population. The levels of Ca125, HE4 and the ROMA results are statistically significant increased in women with ovarian cancer, when they are compared with the cases of benign ovarian cyst or the healthy population. This result can potentially indicate the role of relaxin as tumour marker, similar to ca125. The levels of relaxin, in this study, are statistically significant increased depending on the stage of the disease. Women at FIGO I stage have lower levels of relaxin compared with the levels of relaxin at stage FIGO II, III or IV.

Conclusion* Our study underlines the potential value of relaxin as a diagnostic biomarker in cases of ovarian cancer. Although of limited data, it shows clearly, that high levels of relaxin were consistent in patients with ovarian cancer and the levels are even higher as staging is more severe.

\section{RISK FACTORS FOR RECURRENCE OF BORDERLINE OVARIAN TUMORS AFTER CONSERVATIVE SURGERY: A MULTI CENTER STUDY BY THE FRANCOGYN GROUP}

${ }^{1} \mathrm{~A}$ Ozenne, ${ }^{2} \mathrm{R}$ Emilie, ${ }^{3} \mathrm{~K}$ Yohan, ${ }^{4} \mathrm{C}$ Akladios, ${ }^{5} \mathrm{C}$ Xavier, ${ }^{6} \mathrm{~B}$ Alexandre, ${ }^{7} \mathrm{C}$ Huchon, ${ }^{8} \mathrm{M}$ Camille, 'L Ouldamer*. ${ }^{1}$ Chu Bretonneau Tours, Tours, France, ${ }^{2}$ Hospital Robert Debré, Reims, France; ${ }^{3}$ Chu De Lille, Lille, France; ${ }^{4} \mathrm{CHU}$ de Strasbourg Service des Urgences Adultes, Strasbourg, France; ${ }^{5}$ Marseille University Hospital Timone, Marseille, France; ${ }^{6}$ Hospital Jean Verdier, Bondy, France; ${ }^{7}$ Centre Hospitalier Intercommunal Poissy, Service des urgences, Poissy, France; ${ }^{8}$ Lariboisière Hospital AP-HP, Paris, France

\subsection{6/ijgc-2021-ESG0.519}

Introduction/Background* Introduction: Borderline ovarian tumors (BOT) represent $10-20 \%$ of epithelial tumors of the ovary. Although their prognosis is excellent, the recurrence rate can be as high as $30 \%$, and recurrence in the infiltrative form accounts for $3 \%$ to $5 \%$ of recurrences. Affecting one third of women of childbearing age, the surgical strategy with ovarian conservation is now recommended despite a significant risk of recurrence. Few studies have focused exclusively on patients who have received ovarian conservative treatment in an attempt to identify factors predictive of recurrence and the impact on fertility. The objective of this study was to identify the risk factors for recurrence of BOT after conservative treatment.

Methodology This was a retrospective, multicentre study of women who received conservative surgery for BOT between February 1997 and September 2020. We divided the patients into two groups, the "R group" with recurrence and the "NR group" without recurrence.

Result(s)* Of 175 patients analysed, 35 patients had a recurrence ( $\mathrm{R}$ group, 20\%) and 140 had no recurrence (NR group, 80\%). With a mean follow-up of 30 months (IQ 862.5 ), the overall recurrence rate was $20 \%$. Recurrence was BOT in $17.7 \%(31 / 175)$ and invasive in $2.3 \%(4 / 175)$. The mean time to recurrence was 29.5 months (IQ 16.5-52.5). Initial complete peritoneal staging (ICPS) was performed in $42.5 \%$ of patients $(n=75)$. In multivariate analysis, age at diagnosis, nulliparity, advanced FIGO stage, the presence of peritoneal implants, and the presence of a micropapillary component for serous tumors were factors influencing the occurrence of recurrence. The post-surgery fertility rate was $67 \%$.

Conclusion* This multicentre study is to date one of the largest studies analysing the risk factors for recurrence of BOT after conservative surgery. Five risk factors were found: age at diagnosis, nulliparity, advanced FIGO stage and presence of implants, and a micropapillary component. Only $25 \%$ of the patients with recurrence underwent SPCI. These results 